\title{
Application Value of Whole Exome Sequencing in Screening and Identifying Pathogenic Genes of Hypopharyngeal Carcinoma
}

\author{
Jingwei Yao \\ The Affiliated Nanhua Hospital of University of South China \\ Yubo Ding \\ The Affiliated Nanhua Hospital of University of South China \\ Xiong Liu \\ Nanfang Hospital, Southern Medical University \\ Jialu Huang \\ University of South China \\ Minghui Zhang \\ University of South China \\ Yu Zhang \\ University of South China \\ Yufan Lv \\ The Affiliated Nanhua Hospital of University of South China \\ Zhuoyi Xie \\ University of South China \\ Jianhong Zuo ( $032138414 @ q q . c o m$ ) \\ University of South China
}

\section{Research Article}

Keywords: hypopharyngeal carcinoma, Whole-Exome Sequencing, gene ontology, Gene mutation, pathway analysis

Posted Date: July 6th, 2021

DOI: https://doi.org/10.21203/rs.3.rs-605116/v1

License: (c) (i) This work is licensed under a Creative Commons Attribution 4.0 International License. Read Full License 


\section{Abstract}

Background: Whole-exome sequencing has been used in many cancer research, but it is rarely used in Hypopharyngeal carcinoma. In our research, we performed whole-exome sequencing of DNA from 10 tumor tissue specimens from patients with hypopharyngeal cancer rather than targeted sequencing of specific genes.

Methods: Whole-exome sequencing in 10 patients with hypopharyngeal carcinoma was performed to identify single nucleotide variations (SNVs) and insertions and deletions (INDELs). American College of Medical Genetics and Genomics (ACMG) guidelines were used to evaluate the pathogenicity of the selected variants. Gene Ontology (GO) and pathway enrichment were used to analyze the function and effect of mutated genes. Protein protein interaction (PPI) was analyzed by string online software.

Results: 8113 mutation sites in 5326 genes were identified after strict screening. MEGF8, ITPR1, DYSF, DNAH10, CUL7, MYH14, LRP1, ASTN1, TTN, ASH1L, MYH11, KMT2C were mutated in more than 6 patients. We identified 72 pathogenic or potentially pathogenic mutations in 53 genes according to the ACMG guidelines. GO annotation and KEGG enrichment analysis show the possible effect of these pathogenic genes on cancer.

Conclusion: We identified novel mutations in patients with hypopharyngeal cancer, and provided a foundation for future research on the pathogenesis of hypopharyngeal carcinoma and targeted treatment of hypopharyngeal carcinoma.

\section{Background}

Hypopharyngeal carcinoma is the fourth most common cancer of the head and neck. Most patients with Hypopharyngeal carcinoma have a history of smoking and drinking [1]. Squamous cell carcinoma accounts for the vast majority of pathological types of hypopharyngeal carcinoma, which is characterized by diffuse primary tumor with local dissemination of mucosa and submucosa [2]. Compared with other cancers, such as liver cancer, lung cancer, and other cancers with a higher incidence, there are currently fewer studies on Hypopharyngeal carcinoma. Some of the biomarkers discovered have not yet been put into practice, and the pathogenesis is relatively unclear. Therefore, it is particularly important to find more oncogenes related to the pathogenesis and progress of Hypopharyngeal carcinoma, which will bring benefits for the early diagnosis and clinical treatment of Hypopharyngeal carcinoma. Patients with Hypopharyngeal carcinoma are often accompanied by lymph node metastasis at the time of treatment, which makes it difficult to be treated at present. The current treatment for Hypopharyngeal carcinoma mainly includes surgery, immunotherapy, radiotherapy, chemotherapy, and combined therapy, but the efficacy is not satisfactory, and the five-year survival rate of patients is very low $[3,4]$. Therefore, early diagnosis is particularly important for patients with Hypopharyngeal carcinoma.

In recent years, Next Generation Sequencing (NGS) has brought new breakthroughs to cancer in terms of formulating new cancer biomarkers and discovering mutations $[5,6]$. It mainly includes whole-genome sequencing (WGS) and whole-exome sequencing (WES). Compared with whole-genome sequencing, exons only account for about $1 \%-2 \%$ of the whole genome, and exon mutations are largely related to disease characteristics, WES has higher economic benefits [7, 8]. Whole-exome sequencing plays an important role in identifying gene mutations. Bala et al. found a new tumor suppressor ARID2 in early-onset sporadic rectal cancer through whole-exome sequencing. Patients with ARID2 alterations have poorer survival [9]. In addition, WES has brought new enlightenment for the development of patient treatment plans. PIK3CA and ERBB2 mutations were found in a whole exon sequencing study of cervical carcinomas. The combination of her2 inhibitor neratinib and PIK3CA inhibitor copanlisib has a better tumor regression effect than single inhibitor therapy [10]. Prajish lyer et al. found that KRAS (G12V) mutations may hinder anti-EGFR therapy for gallbladder cancer patients through whole-exome sequencing studies [11]. Plenty of canonical cancer genes have been reported to be related to the development and prognosis of Hypopharyngeal carcinoma in previous studies. These genes are also reported in TCGA and other databases, such as TP53, RAF-1, FHIT, etc. [12-14], but there are still very few studies on Hypopharyngeal carcinoma-related oncogenes, and further mining is needed.

In our research, we performed whole-exome sequencing in ten patients with hypopharyngeal cancer rather than targeted sequencing of specific genes. The sequenced data were compared with the reported genes in databases such as cosmic and

Page 2/16 
TCGA, and some mutations that have not been reported yet were found. In addition, we compared and verified some classic oncogenes reported in the database with the mutations of ten patients.

\section{Methods}

\section{Study population}

10 patients who received surgical treatment in the Affiliated Nanhua Hospital, University of South China from 2016 to 2020 were included in this study. There was no blood relationship between the patients. Ten patients with hypopharyngeal carcinoma were confirmed by pathological biopsy. Our research was approved by the Ethics Committee of the University of South China and complies with the Declaration of Helsinki. All patients agreed to this study and signed an informed consent form.

\section{DNA extraction and gene sequencing}

Qubit 2.0 is used to accurately quantify the concentration of DNA samples. DNA samples with a DNA concentration of $\geq 20 \mathrm{ng} /$ $\mu \mathrm{L}$ and a total amount of $0.6 \mu \mathrm{g}$ or more are used to build the library. Genomic DNA was randomly fragmented into 180-280 bp fragments using a Covaris fragmentation apparatus. The Agilent sureselect human all exon V5 / v6 kit was used for the construction and capture of genomic DNA library. The library with a specific index was hybridized with biotin labeled probe in the liquid phase. Magnetic beads with streptomycin were used to capture the exons, which were linearly amplified by PCR for library quality inspection. Qubit 2.0, Q-PCR, and Agilent 2100 were used to quantify and detect the library.

\section{Sequencing data filtering}

To ensure the quality of information analysis, raw reads are finely filtered to obtain clean reads. The steps of data processing include: (a) removing reads with adapters; (b) Reads in which the proportion of $\mathrm{N}$ more than $10 \%$ is removed ( $\mathrm{N}$ indicates that the nucleobase information cannot be determined); (c) When the number of low-quality (less than 5) bases contained in the single-ended sequencing read exceeds $50 \%$ of the length proportion of the read strip, the pair of paired reads are removed. For those data using double-ended sequencing, we required an average Q30 ratio of above $80 \%$ and an average error rate of below $0.1 \%$.

\section{Data analysis}

The effective sequencing data were compared to the reference genome (human genome) by BWA and samblaster_ B37), and then samblaster was used to mark repeated reads to get the final comparison results. Samtools is used to detect and filter SNP and indel mutations. Annovar is used to annotate the structure and function of the detected variation. We used the annotated and visual database (David) bioinformatics resources 6.8 to identify the biological processes and pathways that 10 patients significantly changed. GO terminology is mainly annotated from GO-CC (cell component), GO-MF (molecular function) and GOBP (biological process). KEGG pathway database is used for pathway enrichment( https://www.kegg.jp/kegg/ ). P $<0.05$ was considered to be statistically significant in the Go annotation and KEGG enrichment analysis. String online software g-db.org/)was used to predict protein-protein interaction (PPI).

\section{Result}

\section{Patient characteristics}

The clinicopathological data of 10 Hypopharyngeal carcinoma patients are summarized in Table 1. The collected clinical data mainly include age, clinical stage, tumor diameter, lymphatic metastasis, distant metastasis, tumor differentiation degree, etc. 
Table 1

The clinicopathological data of 10 Hypopharyngeal carcinoma patients

\begin{tabular}{|c|c|c|}
\hline Variable & & No of patients $\mathrm{N}=10(\%)$ \\
\hline \multirow[t]{2}{*}{ Age (years) } & Mean \pm SD & $55.1 \pm 9.42$ \\
\hline & Range & $35-68$ \\
\hline \multirow[t]{5}{*}{ Clinical stage } & I & $0(0)$ \\
\hline & ॥ & $0(0)$ \\
\hline & III & $7(70)$ \\
\hline & IV & $1(10)$ \\
\hline & NA & $2(20)$ \\
\hline \multirow[t]{5}{*}{ Tumor size } & $\mathrm{T} 1$ & $0(0)$ \\
\hline & $\mathrm{T} 2$ & $4(40)$ \\
\hline & T3 & $1(10)$ \\
\hline & $\mathrm{T} 4$ & $3(30)$ \\
\hline & NA & $2(20)$ \\
\hline \multirow[t]{5}{*}{ Lymph nodes status } & NO & $0(0)$ \\
\hline & $\mathrm{N} 1$ & $3(30)$ \\
\hline & $\mathrm{N} 2$ & $4(40)$ \\
\hline & N3 & $1(10)$ \\
\hline & NA & $2(20)$ \\
\hline \multirow[t]{3}{*}{ Distant metastasis } & Mo & $7(70)$ \\
\hline & M1 & $1(10)$ \\
\hline & NA & $2(20)$ \\
\hline \multirow[t]{3}{*}{ tumor differentiation } & low & $3(30)$ \\
\hline & Medium & $6(60)$ \\
\hline & high & $1(10)$ \\
\hline
\end{tabular}

\section{Gene Variation Spectrum}

The detection of SNV and indel in 10 patients with Hypopharyngeal carcinoma was statistically analyzed. We found that A / G, C / T, G / A transitions were more common than other types of single nucleotide mutations in all patients with Hypopharyngeal carcinoma (Fig. 1. A). Exon mutations accounted for $4.72 \%$ of all mutations. The mutation types of single nucleotide mutations in the exon region were counted. The results showed that missense mutations account for $51.47 \%$ of all exon mutations. Stop gain/loss mutations account for $1.57 \%$ of all mutations, and nonsense mutations account for $45.33 \%$ of all mutations. In addition, there are $1.63 \%$ of unknown mutations (Fig. 1.B). In the detection of insertions and deletions, exon mutations accounted for $1.59 \%$. Frameshift deletion and frameshift insertion accounted for $43.8 \%$ and $11.7 \%$ of all exon mutations, respectively. Nonframeshift deletion and nonframeshift insertion accounted for $32.72 \%$ and $7.01 \%$ of all exon mutations, and stop gain/loss accounted for $1.36 \%$ of all mutations. In addition, there are $3.41 \%$ of unknown mutations (Fig. 1.C). In order to 
better understand the genetic mutations of each patient, the number of SNP/indel in different regions of the genome of each patient and the distribution of the number of different types of SNP/indel in the coding region were counted (Figure S1-S2).

We identified 8113 non-synonymous mutations after screening, including 8096 missense mutations, 1 stop gain mutation and 16 unknown mutations in 5326 genes. There were 1066 mutated genes in two patients, 339 in three patients, 80 in four patients and 22 in five patients. Interestingly, 8 genes including MEGF8, ITPR1, DYSF, DNAH10, CUL7, MYH14, LRP1, and ASTN1 have mutations in six patients, 3 genes including TTN, ASH1L, and MYH11 have mutations in seven patients, and KMT2C has mutations in ten patients. We found that all of the 12 genes had new mutations except kmt2c by comparing the dbSNP database(Table 2, F1, F2).

Table 2

Mutations of mutated genes in at least 6 patients

\begin{tabular}{|lll|}
\hline Gene & Mutation proportion & Mutation information \\
\hline MEGF8 & $60 \%$ & rs377748543,rs370522595,3 novel mutations \\
\hline ITPR1 & $60 \%$ & rs752791333,rs773763162,3 novel mutation \\
\hline DYSF & $60 \%$ & rs573666770,4 novel mutations \\
\hline DNAH10 & $60 \%$ & rs148844278,rs748343428,2 novel mutations \\
\hline CUL7 & $60 \%$ & rs757730802,rs373305024,3 novel mutations \\
\hline TTN & $70 \%$ & rs372496072,rs35683768,rs878903962,rs373854384,rs371908649,3 novel mutations \\
\hline MYH14 & $60 \%$ & rs762779652,rs140118363,4 novel mutations \\
\hline LRP1 & $60 \%$ & rs199726731,9 novel mutations \\
\hline ASTN1 & $60 \%$ & 6 novel mutations \\
\hline ASH1L & $70 \%$ & 6 novel mutations \\
\hline MYH11 & $70 \%$ & rs751495086,rs757099566,4 novel mutations \\
\hline KMT2C & $100 \%$ & rs2479172,rs28522267,rs77735469,rs201062304,rs28522267 \\
\hline
\end{tabular}

\section{High-frequency mutation genes in hypopharyngeal carcinoma in TCGA database}

The 20 oncogenes most frequently mutated in the TCGA database were compared with our screened data, and the results showed that 13 genes including TTN, TP53, ANK3, UPF2, C6, BRCA2, CD163L1, ZNF831, KRT85, MACF1, SYT6, TPO, and SLIT2 had mutations in our samples (Table 3). TTN (70\%), ANK3 (40\%), and TP53 (30\%) have a higher mutation rate, which is also ranked in the top three in the TCGA database. It showed that our results are consistent with the results of the TCGA database. 
Table 3

Comparison of the TOP20 genes of hypopharyngeal carcinoma in the TCGA database and the samples in this research

\begin{tabular}{|c|c|c|c|c|c|c|c|c|c|c|c|}
\hline & 1 & 2 & 3 & 4 & 5 & 6 & 7 & 8 & 9 & 10 & Mutation proportion \\
\hline TTN & - & + & + & - & + & + & + & - & + & + & $70 \%$ \\
\hline TP53 & - & - & + & - & + & - & - & + & - & - & $30 \%$ \\
\hline ANK3 & + & + & - & - & - & + & - & - & + & - & $40 \%$ \\
\hline UPF2 & - & + & - & - & - & - & - & - & - & - & $10 \%$ \\
\hline MFAP3 & - & - & - & - & - & - & - & - & - & - & $0 \%$ \\
\hline DST & - & - & - & - & - & - & - & - & - & - & $0 \%$ \\
\hline C6 & - & + & - & - & - & - & - & - & - & - & $10 \%$ \\
\hline BRCA2 & - & + & - & - & - & - & - & - & - & - & $10 \%$ \\
\hline CD163L1 & - & - & + & - & - & - & - & - & - & - & $10 \%$ \\
\hline MUC16 & - & - & - & - & - & - & - & - & - & - & $0 \%$ \\
\hline ZNF831 & - & - & - & - & - & - & + & - & - & - & $10 \%$ \\
\hline KRT85 & - & - & - & - & - & - & - & - & + & - & $10 \%$ \\
\hline MACF1 & - & - & - & - & - & + & - & + & - & - & $20 \%$ \\
\hline CCDC146 & - & - & - & - & - & - & - & - & - & - & $0 \%$ \\
\hline SYT6 & - & + & - & - & - & - & - & - & - & - & $10 \%$ \\
\hline AN07 & - & - & - & - & - & - & - & - & - & - & $0 \%$ \\
\hline TPO & - & - & - & - & - & - & - & - & + & - & $10 \%$ \\
\hline RBAK-RB & - & - & - & - & - & - & - & - & - & - & $0 \%$ \\
\hline GRM8 & - & - & - & - & - & - & - & - & - & - & $0 \%$ \\
\hline SLIT2 & + & - & - & - & - & - & - & - & + & - & $20 \%$ \\
\hline
\end{tabular}

\section{Screening pathogenic genes based on ACMG guidelines}

72 pathogenic or possibly pathogenic mutations were identified in 53 genes according to the ACMG guidelines, including SNVs or INDELs (Table F3). There were 2 pathogenic or possibly pathogenic mutations in BIVM-ERCC5, FBN2, MYH11, SCN2A, S4CNA and SDHA, 3 pathogenic or possibly pathogenic mutations in RYR1 and SCN5A, and 4 pathogenic or possibly pathogenic mutations in LDLR, TP53 and TTN. 4 unreported mutations were found by comparison to the dbSNP database, and these mutations may cause disease, including two mutations in BIVM-ERCC5 (exon6:c.C640T:p.R214C), (exon14:c. C2002T:p.R668C), GJA3 (exon2:c.C56T:p.T19M), SPG7 (exon9:c.C1198T:p.R400W), these mutations may be related to the pathogenesis of hypopharyngeal carcinoma.

\section{GO annotation of pathogenic and possibly pathogenic genes}

Gene ontology annotation and pathway analyses were performed on 53 pathogenic genes and possibly pathogenic genes. The $\mathrm{BP}$ of these genes is related to muscle contraction, visual perception, cell proliferation, positive regulation of transcription, DNAtemplated, multicellular organism development, sodium ion transmembrane transport, positive regulation of gene expression, 
nervous system development, transport, etc. The main cellular components of these genes involve integral component of membrane, integral component of plasma membrane, mitochondrion, dendrite, intracellular membrane-bounded organelle, $\mathrm{Z}$ disc, mitochondrial matrix, voltage-gated sodium channel complex, apical part of cell, etc. GP-MF annotation showed that these genes are related to some molecular functions, including protein binding, ATP binding, calcium ion binding, calmodulin binding, enzyme binding, protein binding, ubiquitin protein ligase binding, voltage-gated sodium channel activity ATPase activity, coupled to transmembrane movement of substances, flavin adenine dinucleotide binding (Fig. 2, table F4).

\section{Altered pathways}

53 pathogenic or potentially pathogenic genes were analyzed by KEGG enrichment, and the results showed that these genes were highly enriched in some cancers and cancer-related pathways (Table 4). Enrichment pathways mainly include: (a). Various cancers, including thyroid cancer, bladder cancer, endometrial cancer, non-small cell lung cancer, melanoma, pancreatic cancer, colorectal cancer, small cell lung cancer, etc. (b) some signaling pathways closely related to cancer, such as MAPK signaling pathway, HIF-1 signaling pathway, central carbon metabolism in cancer, etc. We constructed a PPI network of 53 pathogenic genes to understand the interaction between 53 pathogenic genes (Fig. 3). The figure shows that these genes include 52 nodes and 62 edges. 
Table 4

Pathway annotation of pathogenic and likely pathogenic genes

\begin{tabular}{|c|c|c|c|c|c|}
\hline ID & Description & Background number & P-Value & count & Gene ID \\
\hline \multirow[t]{2}{*}{ hsa01100 } & \multirow[t]{2}{*}{ Metabolic pathways } & \multirow[t]{2}{*}{1433} & \multirow{2}{*}{$\begin{array}{l}1.21 \times 10^{-} \\
4\end{array}$} & \multirow[t]{2}{*}{9} & \\
\hline & & & & & |NDUFS1|COQ2|PPOX|MUT|SDHA \\
\hline hsa05200 & Pathways in cancer & 530 & $\begin{array}{l}8.35 \times 10^{-} \\
5\end{array}$ & 6 & COL4A5|TP53|JAG1|EGFR|PAX8|AR \\
\hline hsa01522 & Endocrine resistance & 98 & $\begin{array}{l}1.14 \times 10^{-} \\
5\end{array}$ & 4 & TP53|ABCB11|EGFR|JAG1 \\
\hline hsa05165 & $\begin{array}{l}\text { Human papillomavirus } \\
\text { infection }\end{array}$ & 330 & $\begin{array}{l}1.08 \times 10^{-} \\
3\end{array}$ & 4 & TP53|JAG1|COL4A5|EGFR \\
\hline hsa04151 & $\begin{array}{l}\text { PI3K-Akt signaling } \\
\text { pathway }\end{array}$ & 354 & $\begin{array}{l}1.40 \times 10^{-} \\
3\end{array}$ & 4 & TP53|COL4A5|EGFR|INSR \\
\hline hsa02010 & $A B C$ transporters & 45 & $\begin{array}{l}3.84 \times 10^{-} \\
5\end{array}$ & 3 & ABCA4|ABCB11|ABCD1 \\
\hline hsa03420 & Nucleotide excision repair & 47 & $\begin{array}{l}4.34 \times 10^{-} \\
5\end{array}$ & 3 & ERCC2|ERCC5|BIVM-ERCC5 \\
\hline hsa05215 & Prostate cancer & 97 & $\begin{array}{l}3.41 \times 10^{-} \\
4\end{array}$ & 3 & TP53|AR|EGFR \\
\hline hsa05224 & Breast cancer & 147 & $\begin{array}{l}1.11 \times 10^{-} \\
3\end{array}$ & 3 & TP53|JAG1|EGFR \\
\hline hsa04932 & $\begin{array}{l}\text { Non-alcoholic fatty liver } \\
\text { disease (NAFLD) }\end{array}$ & 149 & $\begin{array}{l}1.15 \times 10^{-} \\
3\end{array}$ & 3 & INSR|SDHA|NDUFS1 \\
\hline hsa05160 & Hepatitis C & 155 & $\begin{array}{l}1.29 \times 10^{-} \\
3\end{array}$ & 3 & TP53|LDLR|EGFR \\
\hline hsa05016 & Huntington disease & 193 & $\begin{array}{l}2.38 \times 10^{-} \\
3\end{array}$ & 3 & TP53|SDHA|NDUFS1 \\
\hline hsa04010 & MAPK signaling pathway & 295 & $\begin{array}{l}765 \times 10^{-} \\
3\end{array}$ & 3 & TP53|EGFR|INSR \\
\hline hsa00630 & $\begin{array}{l}\text { Glyoxylate and } \\
\text { dicarboxylate metabolism }\end{array}$ & 30 & $\begin{array}{l}8.63 \times 10^{-} \\
4\end{array}$ & 2 & HOGA1|MUT \\
\hline hsa05216 & Thyroid cancer & 37 & $\begin{array}{l}1.28 \times 10^{-} \\
3\end{array}$ & 2 & TP53|PAX8 \\
\hline hsa05219 & Bladder cancer & 41 & $3^{1.56 \times 10^{-}}$ & 2 & TP53|EGFR \\
\hline hsa00860 & $\begin{array}{l}\text { Porphyrin and chlorophyll } \\
\text { metabolism }\end{array}$ & 42 & $\begin{array}{l}1.63 \times 10^{-} \\
3\end{array}$ & 2 & GUSB|PPOX \\
\hline hsa04913 & Ovarian steroidogenesis & 49 & $\begin{array}{l}2.18 \times 10^{-} \\
3\end{array}$ & 2 & LDLR|INSR \\
\hline
\end{tabular}




\begin{tabular}{|c|c|c|c|c|c|}
\hline ID & Description & Background number & P-Value & count & Gene ID \\
\hline hsa04979 & Cholesterol metabolism & 50 & $\begin{array}{l}2.27 \times 10^{-} \\
3\end{array}$ & 2 & ABCB11|LDLR \\
\hline hsa05213 & Endometrial cancer & 58 & $\begin{array}{l}3.00 \times 10^{-} \\
3\end{array}$ & 2 & TP53|EGFR \\
\hline hsa05223 & $\begin{array}{l}\text { Non-small cell lung } \\
\text { cancer }\end{array}$ & 66 & $\begin{array}{l}3.84 \times 10^{-} \\
3\end{array}$ & 2 & TP53|EGFR \\
\hline hsa05230 & $\begin{array}{l}\text { Central } \\
\text { carbon metabolism in } \\
\text { cancer }\end{array}$ & 69 & $\begin{array}{l}4.18 \times 10^{-} \\
3\end{array}$ & 2 & TP53|EGFR \\
\hline hsa04520 & Adherens junction & 72 & $\begin{array}{l}4.53 \times 10^{-} \\
3\end{array}$ & 2 & EGFR|INSR \\
\hline hsa04976 & Bile secretion & 72 & $\begin{array}{l}4.53 \times 10^{-} \\
3\end{array}$ & 2 & ABCB11|LDLR \\
\hline hsa05218 & Melanoma & 72 & $\begin{array}{l}4.53 \times 10^{-} \\
3\end{array}$ & 2 & TP53|EGFR \\
\hline hsa05212 & Pancreatic cancer & 75 & $\begin{array}{l}4.89 \times 10^{-} \\
3\end{array}$ & 2 & TP53|EGFR \\
\hline hsa05214 & Glioma & 75 & $\begin{array}{l}4.89 \times 10^{-} \\
3\end{array}$ & 2 & TP53|EGFR \\
\hline hsa00983 & $\begin{array}{l}\text { Drug metabolism - other } \\
\text { enzymes }\end{array}$ & 79 & $\begin{array}{l}5.40 \times 10^{-} \\
3\end{array}$ & 2 & GUSB|TK2 \\
\hline hsa05210 & Colorectal cancer & 86 & $\begin{array}{l}6.34 \times 10^{-} \\
3\end{array}$ & 2 & TP53|EGFR \\
\hline hsa04540 & Gap junction & 88 & $\begin{array}{l}6.63 \times 10^{-} \\
3\end{array}$ & 2 & EGFR|TUBB3 \\
\hline hsa04211 & $\begin{array}{l}\text { Longevity regulating } \\
\text { pathway }\end{array}$ & 89 & $\begin{array}{l}6.77 \times 10^{-} \\
3\end{array}$ & 2 & TP53|INSR \\
\hline hsa05410 & $\begin{array}{l}\text { Hypertrophic } \\
\text { cardiomyopathy (HCM) }\end{array}$ & 90 & $\begin{array}{l}6.91 \times 10^{-} \\
3\end{array}$ & 2 & TTN|SGCA \\
\hline hsa05222 & Small cell lung cancer & 93 & $\begin{array}{l}7.35 \times 10^{-} \\
3\end{array}$ & 2 & TP53|COL4A5 \\
\hline hsa05414 & $\begin{array}{l}\text { Dilated cardiomyopathy } \\
\text { (DCM) }\end{array}$ & 96 & $\begin{array}{l}7.81 \times 10^{-} \\
3\end{array}$ & 2 & TTNISGCA \\
\hline hsa04928 & $\begin{array}{l}\text { Parathyroid hormone } \\
\text { synthesis, secretion and } \\
\text { action }\end{array}$ & 106 & $\begin{array}{l}9.41 \times 10^{-} \\
3\end{array}$ & 2 & CASR|EGFR \\
\hline hsa04066 & HIF-1 signaling pathway & 109 & $\begin{array}{l}9.92 \times 10^{-} \\
3\end{array}$ & 2 & EGFR|INSR \\
\hline
\end{tabular}

\section{Discussion}


Hypopharyngeal carcinoma is relatively rare compared with other cancers, accounting for about $3 \%$ of head and neck malignant tumors, and most patients are already in the advanced stage when they are diagnosed $[15,16]$. At present, there are few studies on the mechanism of Hypopharyngeal carcinoma. In order to better treat Hypopharyngeal carcinoma, ten patients with Hypopharyngeal carcinoma were subjected to whole-exome sequencing rather than targeted sequencing of specific genes, aiming to discover more mutations related to the occurrence and development of Hypopharyngeal carcinoma.

8113 mutation sites were found in 5326 genes after strict screening conditions. And we found that MEGF8, ITPR1, DYSF, DNAH10, CUL7, MYH14, LRP1, ASTN1, TTN, ASH1L, and MYH11 mutated in at least 6 patients, while KMT2C mutated in 10 patients. To verify the accuracy of our results in this study, our screened data were compared with the TOP20 gene in the TCGA database, and we found that the top three genes (TTN, ANK3, and TP53) in the Hypopharyngeal carcinoma mutation genes in the TCGA database also had mutations in more patients in our samples, which to some extent proved the accuracy of our results.

In order to determine the pathogenicity of the selected genes, 72 mutations in 53 genes were selected according to the international ACMG guidelines. We found that two pathogenic or possibly pathogenic mutation sites in BIVM-ERCC5, FBN2, MYH11, SCN2A, S4CNA and SDHA, three pathogenic or possibly pathogenic mutation sites in RYR1 and SCN5A, and four pathogenic or possibly pathogenic mutation sites in LDLR, TP53 and TTN. In addition, we found four sites not reported in dbSNP database,including two mutations in BIVM-ERCC5 (exon6: c.c640t: p.r214c), (exon14: c.c2002t: p.r668c), GJA3 (Exon2: c.c56t: p.t19m) and SPG7 (exon9: c.c1198t: p.r400w), which may be related to the occurrence and development of hypopharyngeal carcinoma.

To further confirm the role of these causative genes and whether they are associated with the pathogenesis of Hypopharyngeal carcinoma, GO annotation, KEGG enrichment analysis, and PPI network were constructed. The results show that many of these genes are associated with cancer. However, Hypopharyngeal carcinoma is a small cancer species, so there are few reports about Hypopharyngeal carcinoma in the database, which is also one of the significance of our study. Interestingly, we found that TTN, MYH11, SDHA, and RYR1 have mutations in many samples, and they have more than one disease-causing mutation in 10 patients. Interestingly, we found that TTN, MYH11, SDHA, and RYR1 had mutations in many samples in our research, which also had more than one pathogenic mutation site in 10 patients.

KMT2C, a member of histone methyltransferase (H3K4ME3), is a kind of chromotin modifying and remodelling protein [17]. KMT2C can catalyze the methylation of protein sites to change the structure of chromosomes and finally affect the transcription process of target genes $[18,19]$. Previous studies have shown that KMT2C is mutated in a variety of cancers, including osteosarcoma, acute myeloid leukemia, breast cancer, and gastric cancer [20-23]. However, there are very few reports about the relationship between KMT2C mutation and hypopharyngeal carcinoma. In our research, we found that all samples had KMT2C mutation after strict screening of the whole-exome sequencing results of ten patients, which may indicate the relationship between $\mathrm{KMT} 2 \mathrm{C}$ and the pathogenesis of Hypopharyngeal carcinoma, and provide thinking for future research of the pathogenesis of Hypopharyngeal carcinoma.

The protein encoded by MYH11 is a smooth muscle myosin belonging to the myosin heavy chain family, which acts as a contractile protein by participating in the hydrolysis of adenosine triphosphate [24]. There have been some reports on the effect of MYH11 on cancer. Studies have confirmed that it is related to the pathogenesis or prognosis of lung cancer, acute myeloid leukemia, gastric cancer, colorectal cancer and breast cancer [24-27]. However, the relationship between MYH11 and Hypopharyngeal carcinoma has not yet been reported. In our research, we found that MYH11 has mutations in 7 patients, and we found two pathogenic or possibly pathogenic mutations (rs375159635, rs751495086), which may be related to the pathogenesis of Hypopharyngeal carcinoma. In addition, SDHA is mainly related to gangliomas [28-30], RYR1 is mainly related to myopathy [31,32], and TTN is mainly related to dilated cardiomyopathy [33,34]. Interestingly, we found that these three genes have mutations in more than five patients, and all of them have two or more pathogenic or possibly pathogenic mutation sites, which indicates their potential in the pathogenesis of Hypopharyngeal carcinoma.

Inevitably, our research also has limitations. The first is that our sample is not large enough, which can provide a direction for the research of the mechanism, but subsequent experiments with a large sample are needed to confirm the results. In addition, it 
is not enough to analyze the potential function and pathway of genes through bioinformatics, and later experiments are necessary.

\section{Conclusion}

We performed whole-exome sequencing on 10 patients with Hypopharyngeal carcinoma and screened out some genes that may be related to the pathogenesis of Hypopharyngeal carcinoma, including a great number of novel mutations that have not been reported. GO annotation, KEGG enrichment analysis, and PPI network were constructed for subsequent pathogenicity and pathway analysis. Our research has deepened the understanding of the pathogenesis of hypopharyngeal carcinoma and provided a foundation for subsequent research.

\section{Abbreviations}

WGS: whole-genome sequencing

WES: whole-exome sequencing

SNV: single nucleotide variations

INDEL: insertions and deletions

ACMG: American College of Medical Genetics and Genomics

GO: Gene Ontology

PPI: protein-protein interaction

\section{Declarations}

\section{Ethics approval and consent to participate}

Our study was approved by the ethics committee of Affiliated Nanhua Hospital, University of South China. Informed consent was obtained from all individual participants in the study.

\section{Consent for publication}

Not applicable.

\section{Availability of data and materials}

The datasets used and/or analysed during the current study are available from the corresponding author on reasonable request.

\section{Competing interests}

The authors have no conflicts of interest to declare.

\section{Funding}

This work is supported by the Key Research Program from the Science and Technology Department of Ningxia Hui Autonomous Region, China (2019BFH02012); the Key Research Program of Hunan Health Committee (20201909); the Program of Hengyang science and Technology Bureau (2017-1, 2020-67);

\section{Authors' contributions}


The study was carried out in collaboration with all the authors. Yao Jingwei and Zuo Jianhong carried out genetic research and drafted the manuscript. Ding Yubo and Huang Jialu designed the experiments and methods. Xiong Liu, Zhang Minghui, Zhang Yu, Lv Yufan and Xie Zhuoyi collected samples and patient information. All authors read and approved the final manuscript.

\section{Acknowledgments}

We thank all the authors for their contributions to this research. We also thank all participants who provided blood samples.

\section{References}

1. Zeng J, Tang Y, Wu P, Fang X, Wang W, Fan Y, et al. Alcohol consumption, tobacco smoking, betel quid chewing and oral health associations with hypopharyngeal cancer among men in Central South China: a case-control study. Cancer Manag Res. 2019;11:6353-64.

2. Piazza C, Paderno A, Ravanelli M, Pessina C. Clinical and Radiological Evaluation of Hypopharyngeal Carcinoma. Adv Otorhinolaryngol. 2019;83:35-46.

3. Tsai YT, Chen WC, Chien CY, Hsu CM, Lee YC, Tsai MS, et al. Treatment patterns and survival outcomes of advanced hypopharyngeal squamous cell carcinoma. World J Surg Oncol. 2020;18(1):82.

4. Eckel HE, Bradley PJ. Natural History of Treated and Untreated Hypopharyngeal Cancer. Adv Otorhinolaryngol. 2019;83:2734.

5. Morganti S, Tarantino P, Ferraro E, D'Amico P, Duso BA, Curigliano G. Next Generation Sequencing (NGS): A Revolutionary Technology in Pharmacogenomics and Personalized Medicine in Cancer. Adv Exp Med Biol. 2019;1168:9-30.

6. Mosele F, Remon J, Mateo J, Westphalen CB, Barlesi F, Lolkema MP, et al. Recommendations for the use of next-generation sequencing (NGS) for patients with metastatic cancers: a report from the ESMO Precision Medicine Working Group. Ann Oncol. 2020;31(11):1491-505.

7. Meienberg J, Zerjavic K, Keller I, Okoniewski M, Patrignani A, Ludin K, et al. New insights into the performance of human whole-exome capture platforms. Nucleic Acids Res. 2015;43(11):e76.

8. Rabbani B, Tekin M, Mahdieh N. The promise of whole-exome sequencing in medical genetics. J Hum Genet. 2014;59(1):515.

9. Bala P, Singh AK, Kavadipula P, Kotapalli V, Sabarinathan R, Bashyam MD. Exome sequencing identifies ARID2 as a novel tumor suppressor in early-onset sporadic rectal cancer. Oncogene. 2021;40(4):863-74.

10. Zammataro L, Lopez S, Bellone S, Pettinella F, Bonazzoli E, Perrone E, et al. Whole-exome sequencing of cervical carcinomas identifies activating ERBB2 and PIK3CA mutations as targets for combination therapy. Proc Natl Acad Sci U S A. 2019;116(45):22730-6.

11. Iyer P, Shrikhande SV, Ranjan M, Joshi A, Gardi N, Prasad R, et al. ERBB2 and KRAS alterations mediate response to EGFR inhibitors in early stage gallbladder cancer. Int J Cancer. 2019;144(8):2008-19.

12. Sun J, Lin L, Zhang J, Hu C, Wang J. The prognostic value of USP7 and p53 in advanced hypopharyngeal carcinoma. Ann Diagn Pathol. 2021;51:151695.

13. Li Y, Lu T, Hu G. Gene sequencing and expression of Raf-1 in lymphatic metastasis of hypopharyngeal carcinoma. Cancer Biomark. 2020;28(2):181-91.

14. Wu H, Luan X, Pan X, Huang J, Wang Q, Lei D. [Mutation and abnormal expression of FHIT gene in hypopharyngeal carcinoma]. Lin Chung Er Bi Yan Hou Tou Jing Wai Ke Za Zhi. 2009;23(6):245-8, 53.

15. Li L, Wang Z, Lu T, Li Y, Pan M, Yu D, et al. Expression and Functional Relevance of ANXA1 in Hypopharyngeal Carcinoma with Lymph Node Metastasis. Onco Targets Ther. 2021;14:1387-99.

16. Kwon DI, Miles BA, Education Committee of the American H, Neck S. Hypopharyngeal carcinoma: Do you know your guidelines? Head Neck. 2019;41(3):569-76.

17. Chiappetta C, Carletti R, Della Rocca C, Di Cristofano C. KMT2C modulates migration and invasion processes in osteosarcoma cell lines. Pathol Res Pract. 2019;215(10):152534. 
18. Rao RC, Dou Y. Hijacked in cancer: the KMT2 (MLL) family of methyltransferases. Nat Rev Cancer. 2015;15(6):334-46.

19. Bannister AJ, Kouzarides T. Regulation of chromatin by histone modifications. Cell Res. 2011;21(3):381-95.

20. Bowler TG, Pradhan K, Kong Y, Bartenstein M, Morrone KA, Sridharan A, et al. Misidentification of MLL3 and other mutations in cancer due to highly homologous genomic regions. Leuk Lymphoma. 2019;60(13):3132-7.

21. Chen X, Zhang G, Chen B, Wang Y, Guo L, Cao L, et al. Association between histone lysine methyltransferase KMT2C mutation and clinicopathological factors in breast cancer. Biomed Pharmacother. 2019;116:108997.

22. Zhang G, Zhou S, Zhong W, Hong L, Wang Y, Lu S, et al. Whole-Exome Sequencing Reveals Frequent Mutations in Chromatin Remodeling Genes in Mammary and Extramammary Paget's Diseases. J Invest Dermatol. 2019;139(4):789-95.

23. Cho SJ, Yoon C, Lee JH, Chang KK, Lin JX, Kim YH, et al. KMT2C Mutations in Diffuse-Type Gastric Adenocarcinoma Promote Epithelial-to-Mesenchymal Transition. Clin Cancer Res. 2018;24(24):6556-69.

24. Jo YS, Kim MS, Yoo NJ, Lee SH. Somatic Mutations and Intratumoral Heterogeneity of MYH11 Gene in Gastric and Colorectal Cancers. Appl Immunohistochem Mol Morphol. 2018;26(8):562-6.

25. Biernacki MA, Foster KA, Woodward KB, Coon ME, Cummings C, Cunningham TM, et al. CBFB-MYH11 fusion neoantigen enables T cell recognition and killing of acute myeloid leukemia. J Clin Invest. 2020;130(10):5127-41.

26. Nie MJ, Pan XT, Tao HY, Xu MJ, Liu SL, Sun W, et al. Clinical and prognostic significance of MYH11 in lung cancer. Oncol Lett. 2020;19(6):3899-906.

27. Alhopuro P, Karhu A, Winqvist R, Waltering K, Visakorpi T, Aaltonen LA. Somatic mutation analysis of MYH11 in breast and prostate cancer. BMC Cancer. 2008;8:263.

28. Huang YC, Chang $\mathrm{HH}$, Chen MH, Huang KH, Li AF, Lin $\mathrm{CH}$, et al. Somatic SDHA mutations in paragangliomas in siblings: Case report of 2 cases. Medicine (Baltimore). 2020;99(41):e22497.

29. van der Tuin K, Mensenkamp AR, Tops CMJ, Corssmit EPM, Dinjens WN, van de Horst-Schrivers ANA, et al. Clinical Aspects of SDHA-Related Pheochromocytoma and Paraganglioma: A Nationwide Study. J Clin Endocrinol Metab. 2018;103(2):43845.

30. Tufton N, Ghelani R, Srirangalingam U, Kumar AV, Drake WM, lacovazzo D, et al. SDHA mutated paragangliomas may be at high risk of metastasis. Endocr Relat Cancer. 2017;24(7):L43-L9.

31. Zullo A, Perrotta G, D'Angelo R, Ruggiero L, Gravino E, Del Vecchio L, et al. RYR1 Sequence Variants in Myopathies: Expression and Functional Studies in Two Families. Biomed Res Int. 2019;2019:7638946.

32. Jokela M, Tasca G, Vihola A, Mercuri E, Jonson PH, Lehtinen S, et al. An unusual ryanodine receptor 1 (RYR1) phenotype: Mild calf-predominant myopathy. Neurology. 2019;92(14):e1600-e9.

33. Hirayama-Yamada K, Inagaki N, Hayashi T, Kimura A. A Novel Titin Truncation Variant Linked to Familial Dilated Cardiomyopathy Found in a Japanese Family and Its Functional Analysis in Genome-Edited Model Cells. Int Heart J. 2021;62(2):359-66.

34. Ishihara M, Nakanishi N, Tsutsumi R, Hara K, Machida K, Yamamoto N, et al. Elevated Urinary Titin and its Associated Clinical Outcomes after Acute Stroke. J Stroke Cerebrovasc Dis. 2021;30(3):105561.

\section{Figures}


A

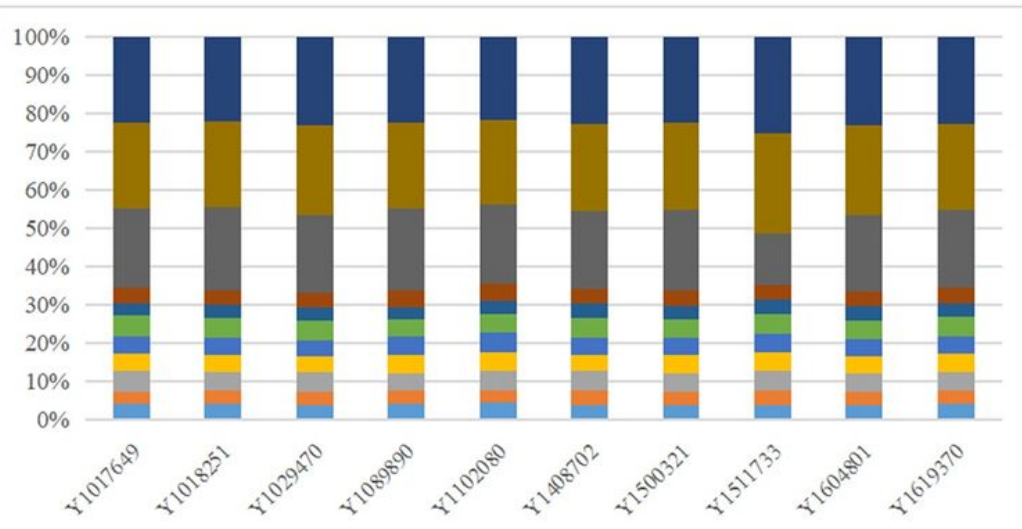

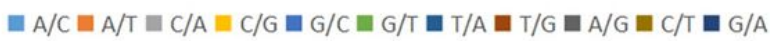

B

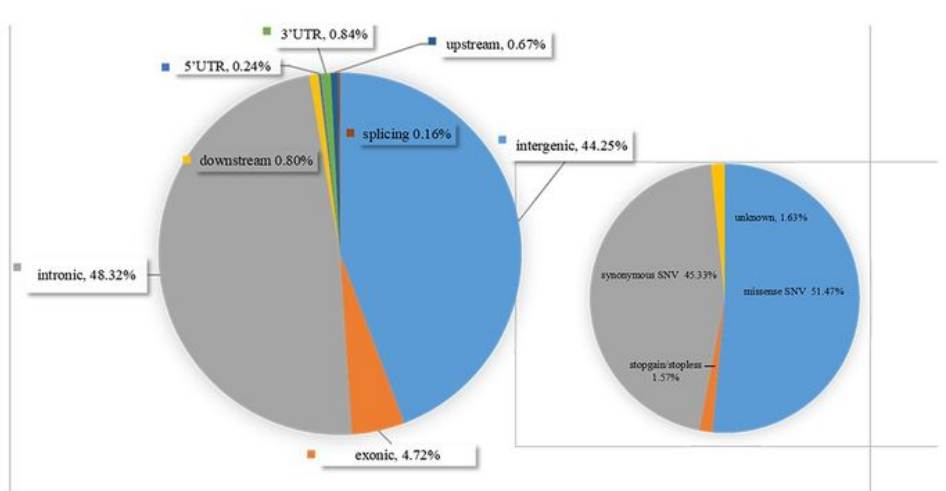

C

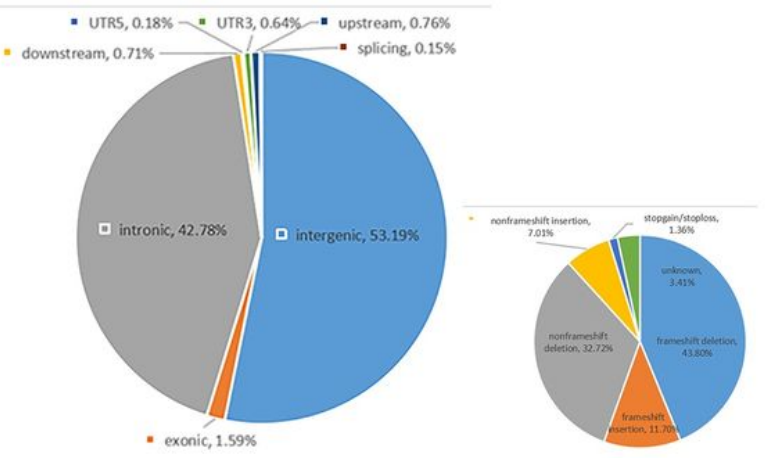

\section{Figure 1}

Targeted sequencing of 10 patients with hypopharyngeal carcinoma A. The proportion of nucleotide mutations in 10 patients with hypopharyngeal carcinoma B. The proportion of mutation in different regions in SNV and the proportion of different mutation type in exon regions $\mathrm{C}$. The proportion of mutation in different regions in indel and the proportion of different mutation type in exon regions 

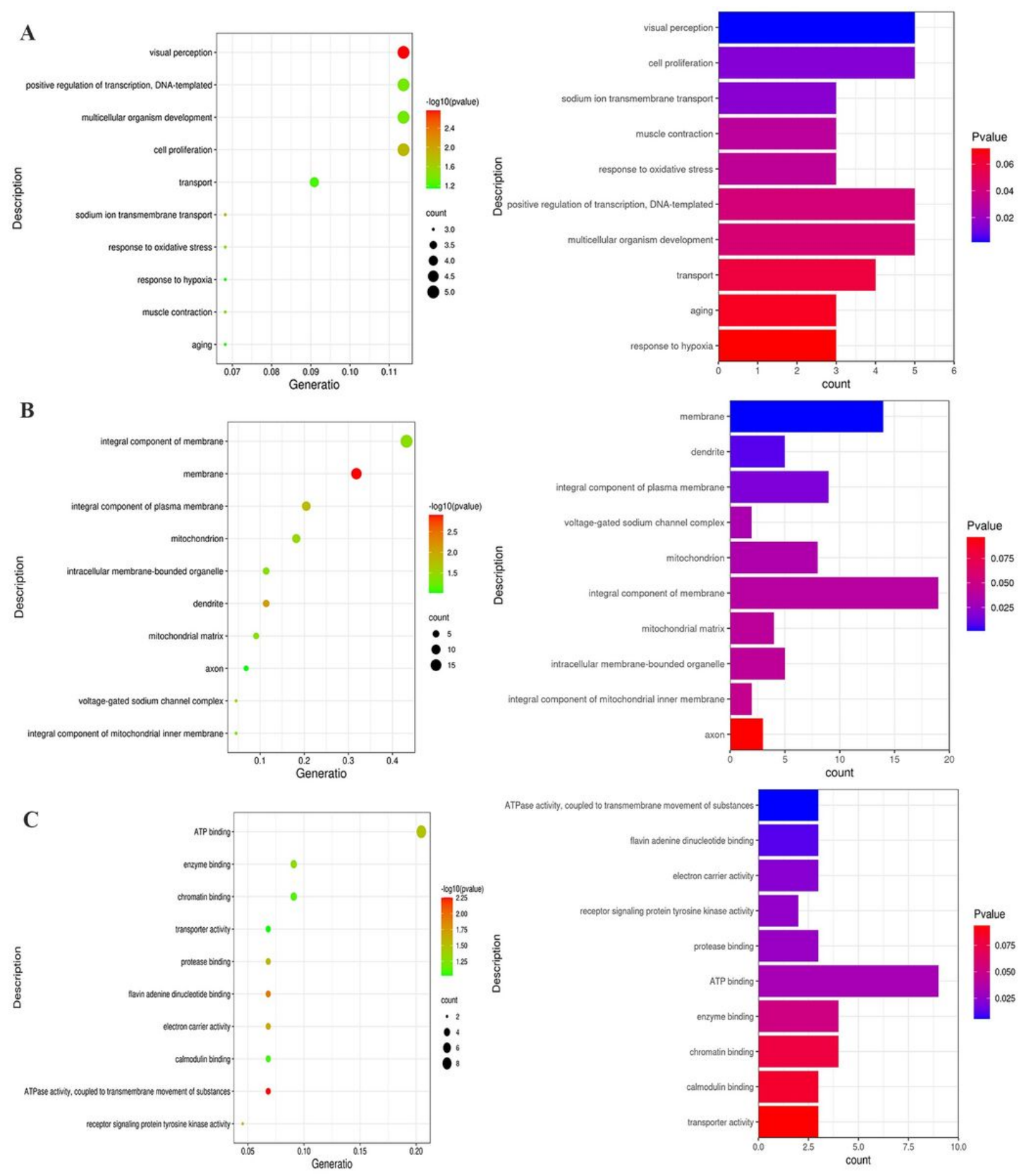

Figure 2

TOP 10 in Go enrichment analysis A. Biological process (GO-BP) of 53 pathogenic and likely pathogenic genes B. Cellular component (GO-CC) of pathogenic and likely pathogenic genes $\mathrm{C}$. Molecular function (GO-MF) of pathogenic and likely pathogenic genes 


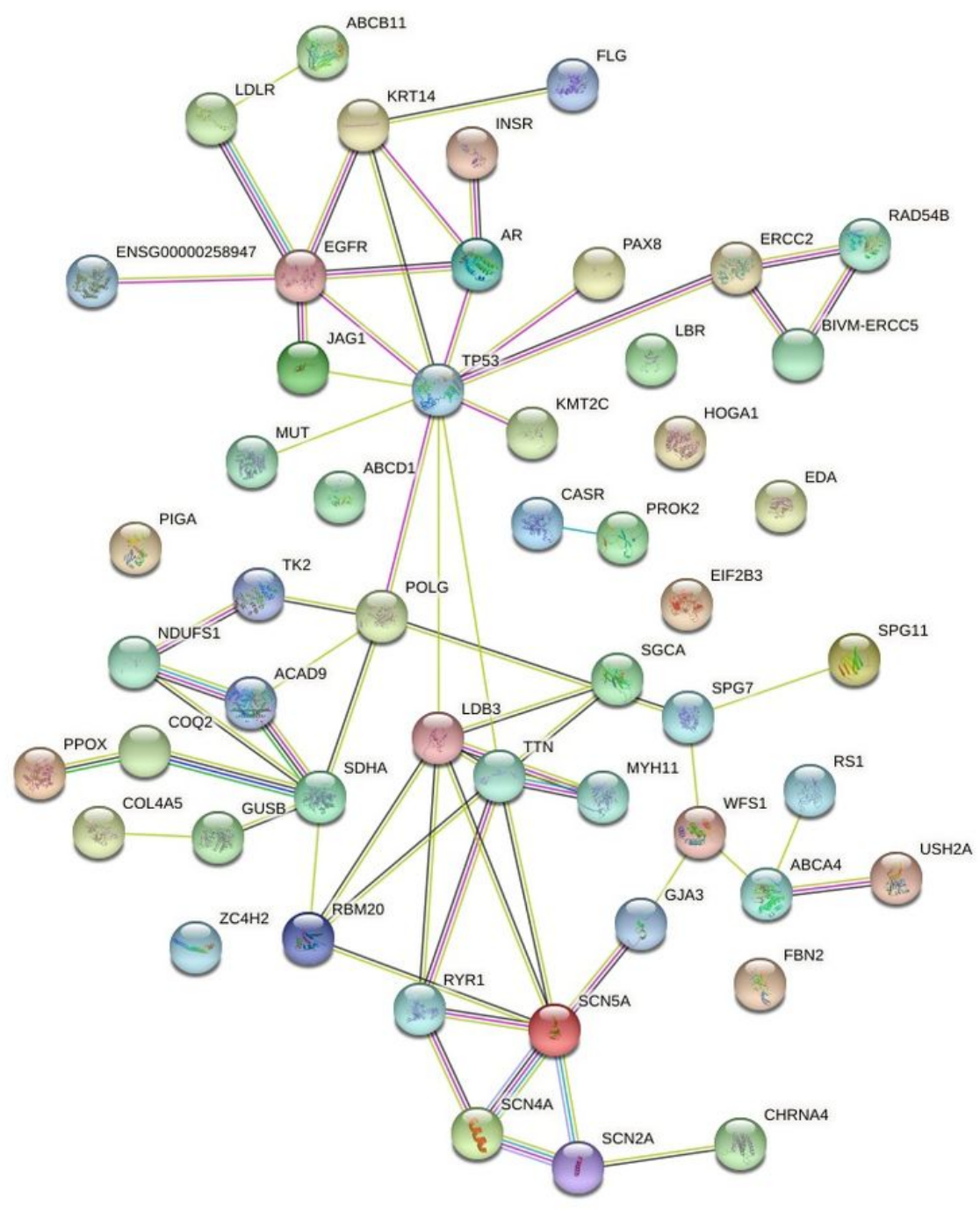

Figure 3

PPI network of 53 pathogenic or possibly pathogenic genes

\section{Supplementary Files}

This is a list of supplementary files associated with this preprint. Click to download.

- Additionalfile1Table.docx

- Additionalfile2Fig.S1.tif

- Additionalfile2Fig.S2.tif

- Additionalfile2Figure.docx 